\title{
Pathophysiological studies of neuromuscular function in subacute organophosphate poisoning induced by phosmet
}

\author{
J L Good, R K Khurana, R F Mayer, W M Cintra, E X Albuquerque
}

\begin{abstract}
A 51 year old man developed progressive cranial and proximal muscle weakness, hyperreflexia and mental change. The disorder progressed over 9 days following the fifth weekly spraying with the organophosphate (OP) insecticide, phosmet, with limited symptoms of acute toxicity. Marked decremental responses of $50-80 \%$ on slow and fast rates of stimulation were improved to $15 \%$ by edrophonium or neostigmine. Intracellular recordings at the endplate region of intercostal muscle revealed small miniature endplate potentials (mepps), reduced mean acetylcholine sensitivity and normal membrane potentials. Electronmicroscopy revealed degeneration and regeneration of the endplates. This study demonstrates that OP poisoning due to phosmet can produce a subacute postsynaptic neuromuscular syndrome without marked symptoms of acute toxicity.
\end{abstract}

(F Neurol Neurosurg Psychiatry 1993;56:290-294)

University of

Maryland School of Medicine, Baltimore, Maryland, USA Department of Neurology

J L Good

R K Khurana

R F Mayer

Department of

Pharmacology and

Experimental

Therapeutics

E X Albuquerque

Laboratory of

Molecular

Pharmacology II,

Institute of Biophysics

"Carlos Chagas

Filho", Federal

University of Rio De

Janeiro, Rio De

Janeiro, Brazil

W M Cintra

Correspondence to:

Dr Good, Department of

Neurology, Room N4W46,

University of Maryland

University of Maryland

Hospital, 22 S Greene

Street, Baltim
21201, USA

Received 17 December 1991 and in revised form

11 March 1992

Accepted 16 March 1992 suggested a neuromuscular junction (NMJ) defect of the postsynaptic type, but the exact mechanism of the dysfunction was not determined. ${ }^{10}$

We describe the pathophysiological changes in a patient who presented with a subacute progressive neuromuscular syndrome without marked symptoms of acute toxicity after multiple OP insecticide sprayings. The defect in this neuromuscular syndrome occurred at the endplate-receptor sites, is similar to the intermediate syndrome previously reported, ${ }^{10}$ and differs from the acute and delayed syndromes. ${ }^{1}$ These results are important in the diagnosis and management of patients exposed to organophosphates. A preliminary report of this study has been published in abstract form. ${ }^{11}$

\section{Case report}

A 51 year old man was admitted to the hospital 5 days after the onset of diplopia, light headedness and a staggering gait. The patient developed these symptoms 18 hours after the fifth weekly exposure to a liquid spray insecticide containing an organophosphate compound, phosmet, (phosphorodiothioic acid S-[(1,3-dihydro-1,3-dioxo-2H-isoindol-2-yl) methyl] 0,0dimethylester). He described his face and hands becoming wet from the insecticide and he did not bathe until the following morning. Over the next few days, he noted progressive unsteadiness of gait, dysphagia, change in voice tone and excessive diaphoresis. Five days after exposure, he noted jaw weakness and droopy eyelids.

The patient's general examination was normal. Profuse oral secretions, bilateral ptosis, and marked facial diaphoresis were noted. He was alert, mentation was normal, and speech was dysarthric. Extraocular movements were limited in all directions with the exception of downgaze. Pupils were $3 \mathrm{~mm}$ and reactive to light. There was moderate symmetrical weakness of facial, jaw, tongue and neck muscles. There was mild weakness of distal muscles and marked weakness of proximal muscles, especially antigravity muscles that fatigued. The tendon jerks were brisk throughout. Bilateral Babinski responses were present. Sensory examination was normal.

The patient was admitted with the presumed 
diagnosis of acute OP poisoning. Atropine ( $2 \mathrm{mg}$ ) and pralidoxime chloride (2-PAM, $1 \mathrm{gm})$ infusions were started. Within several minutes of 2-PAM infusion, the patient had increased systemic and ventilatory weakness requiring intubation. After the patient was stabilised on a ventilator, 3 additional doses of 2-PAM were given without side effects over the next 24 hours. Atropine $(1.0 \mathrm{mg}$ initially and thereafter $0.5 \mathrm{mg}$ ) was continued 4 times daily for 6 days. Paralysis was maximum on day 9 . At this time, he was observed to have visual hallucinations, disorientation, and myoclonic jerks of his extremities. These signs persisted for approximately 10 days during which time the EEC showed diffuse slow wave activity.

The patient remained unchanged for the following 18 days, and supportive care continued. By day 28, there was improvement in strength of neck flexion, shoulder and antigravity muscles. By day 44 , he no longer required mechanical ventilation or tube feedings. Babinski responses were absent. Examination on day 64 (time of the muscle biopsy) showed that he had mild fatiguable proximal and antigravity muscle weakness in the upper and lower limbs. Facial and oropharyngeal movements were near normal. There was mild subjective diplopia on extreme lateral gaze. Neuromuscular examination was normal by 5 months.

Laboratory studies revealed normal routine blood and urine studies, creatine kinase, EKG and chest radiographs. MRI of the brain was normal. CSF was normal except for a total protein of $75 \mathrm{mg} \%$. On admission red blood cell cholinesterase level was within normal limits (6110 U/L); and follow up levels on days 16 and 23 were 5420 and 4180 respectively and at 10 months ( $5114 \mathrm{U} / \mathrm{L})$ remained normal (normal range 6666-3590). Studies to rule out other causes of neuromuscular disease were normal. ANA and acetylcholine receptor antibody titres were negative.

\section{Methods}

Nerve conduction and repetitive stimulationneuromuscular studies were performed in peripheral nerves of upper and lower extremities using established techniques. ${ }^{12}{ }^{13}$ Recordings were performed in the intensive care unit

Table 1 Nerve conduction and repetitive stimulation studies in median nerve after phosmet exposure

\begin{tabular}{|c|c|c|c|c|c|c|c|}
\hline \multirow{3}{*}{$\begin{array}{l}\text { Time } \\
\text { Days }\end{array}$} & \multirow{3}{*}{$\begin{array}{l}C M A P \\
m V\end{array}$} & \multirow{3}{*}{$\begin{array}{l}D L \\
M S\end{array}$} & \multirow{3}{*}{$\begin{array}{l}M C V \\
M / S\end{array}$} & \multicolumn{4}{|c|}{$\%$ Decrement at $5 \mathrm{~Hz}$} \\
\hline & & & & \multicolumn{2}{|l|}{ Before } & \multicolumn{2}{|c|}{ After Tensilon } \\
\hline & & & & $4 \operatorname{th} R$ & $9 \operatorname{th} R$ & 4 th $R$ & $9 \operatorname{th} R$ \\
\hline $\begin{array}{r}9 \\
11 \\
12 \\
19 \\
32 \\
47 \\
95\end{array}$ & $\begin{array}{r}9.0 \\
11.6 \\
13.0 \\
19.0 \\
18.0 \\
22.0 \\
19.0\end{array}$ & $\begin{array}{l}4 \cdot 7 \\
4 \cdot 2 \\
4 \cdot 2 \\
3 \cdot 9\end{array}$ & $\begin{array}{l}56 \\
53 \\
62 \\
55\end{array}$ & $\begin{array}{r}48 \\
55 \\
58 \\
42 \\
39 \\
16 \\
+6\end{array}$ & $\begin{array}{r}43 \\
43 \\
46 \\
26 \\
27 \\
9 \\
+6\end{array}$ & $\begin{array}{r}33 \\
10 \\
7 \\
16 \\
2\end{array}$ & $\begin{array}{r}19 \\
7 \\
0 \\
6 \\
2\end{array}$ \\
\hline \multicolumn{2}{|c|}{$\begin{array}{l}\text { Normal subjects } \\
\text { Mean (SD) } 17 \cdot 0(4 \cdot 8)\end{array}$} & $3.5(0.5)$ & $55(4)$ & $0(10)$ & $0(10)$ & $0(10)$ & $0(10)$ \\
\hline
\end{tabular}

and care was taken to keep the patient warm (rectal temperature of $37^{\circ} \mathrm{C}$ ) during the procedure. Recordings were obtained at 9 to 95 days after exposure to phosmet.

Repetitive stimulation was performed at rates of $2,5,10,20$, and $50 \mathrm{~Hz}$ for 9 responses in the median and peroneal nerves. Recordings of the median nerve were made before and after $10 \mathrm{mg}$ of intravenous edrophonium at days 11 to 47 . Recordings were made 5 to 40 minutes after $1.5 \mathrm{mg}$ of intramuscular neostigmine on day 12. Measurements of the motor amplitudes were peak-to-peak and percentage change (decrement) was calculated for the fourth and ninth responses compared with the initial one.

Concentric needle and single fibre (SF EMG) electromyography and fibre densities were performed in the extensor digitorum communis and first dorsal interosseus muscles using established techniques. ${ }^{14}$ SF EMG recordings were obtained when the patient was improving and able to cooperate, 3-4 months after exposure.

A diagnostic intercostal muscle biopsy was obtained 2 months after exposure because of persistent muscle weakness with informed consent by the patient. Muscle was removed from the right sixth intercostal space in the anterior axillary line using standardised aneasthesia and surgical technique. The muscle was immediately placed in oxygenated KrebsRinger solution and prepared for intracellular recordings and ultrastructure as previously described. ${ }^{15}$ Conventional microelectrode techniques were used for recording from human intercostal muscle. High resistance pipettes (approximately $150 \mathrm{M} \Omega$ ) filled with 2.5 M acetylcholine (ACh) were used for microiontophoretic application of $\mathrm{ACh}$ at the neuromuscular junctions. Results were compared statistically (Student $t$ test) with control data that we have obtained from normal adult human intercostal muscle biopsied during thoracotomies for cardiopulmonary disease. The muscle fibres were fixed in ice-cold phosphate buffered $2 \cdot 5 \%$ glutaraldehyde for thin sectioning and electron microscopic examination of the endplate regions. ${ }^{15} 16$

\section{Results}

\section{Clinical electrophysiology}

Initial recordings were performed 9 days after exposure at which time the weakness was maximal. Electromyography at this time did not show any spontaneous activity such as fasciculations or fibrillation potentials. In median, ulnar and peroneal nerves, distal motor responses were mildly reduced in amplitude and prolonged in latency. Motor conduction velocities were normal. No nerve conduction block on proximal or distal stimulation or repetitive motor responses were observed following single supramaximal shocks. Antidromic motor $F$ responses were normal in amplitude and latency. Serial studies were performed in the median nervethenar muscles and these results are shown in table 1 . 
Irigure 1 Recordings of compound muscle action potentials from the thenar muscles on day 9 using surface electrodes stimulating the median nerve at the wrist at rates of $50 \mathrm{~Hz}(A), 20 \mathrm{~Hz}(B)$ and $2 \mathrm{~Hz}\left(C^{\prime}\right)$. Note the marked decrement by the fourth response with some recovery by the last response at rates of 2 and $20 \mathrm{~Hz}$ but not at $50 \mathrm{~Hz}$. Amplitude calibration is $2 \mathrm{mV}$.

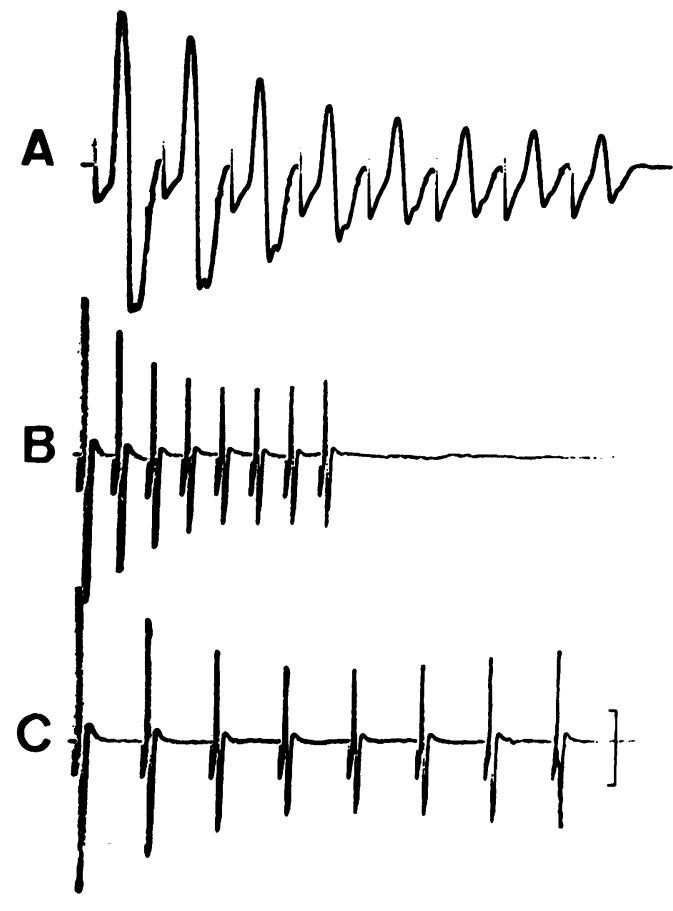

Repetitive stimulation at slow rates $(2-10 \mathrm{~Hz})$ in the median and peroneal nerves produced marked decremental responses $(44-50 \%)$ at the fourth response with some recovery by the last response (figure 1 ). At fast rates of stimulation $(50 \mathrm{~Hz})$ marked decrement $(80 \%)$ was observed without recovery. Serial recordings were performed in the median nerve and the results are shown in table 1. Response to intravenous edrophonium chloride was studied from days 11 to 47 (table 1) and showed a marked decrease in the decrement $(58-42 \%$ reduced to $33-7 \%$ ) at slow and fast rates of stimulation. The decrease in decrement was associated with transient increase in strength on muscle testing. The beneficial effect of edrophonium persisted throughout the 6 week period of neuromuscular weakness. Similar beneficial effects were obtained with intramuscular neostigmine that reduced the decremental response of $58 \%$ (4th response at $65 \mathrm{~Hz}$ ) to $10 \%$ at 35 minutes after the injection.

Three months after exposure when the patient had mild proximal muscle weakness and fatiguability, no decremental responses were observed. However, SF EMG revealed increased variability in the interpotential intervals (jitter) in all 20 potential pairs examined.

Table 2 Endplate recordings in intercostal muscles in patient with phosmet exposure and normal subjects

\begin{tabular}{|c|c|c|}
\hline Recordings & Patient & Normals \\
\hline $\begin{array}{l}\text { Resting membrane } \\
\text { Potential, mV } \\
\text { MEPP } \\
\text { Amplitude, mV } \\
\text { MEPP } \\
\text { Frequency, Sec }{ }^{-1} \\
\text { ACh sensitivity } \\
\mathrm{mV} / \mathrm{nC}\end{array}$ & $\begin{array}{l}-75(2 \cdot 73) \\
45^{\mathrm{a}} \\
0 \cdot 56(0.07)^{\star} \\
40 / 481^{\mathrm{h}} \\
0 \cdot 25(0 \cdot 02)^{\star} \\
40 / 581 \\
1182(216)^{\star} \\
11\end{array}$ & $\begin{array}{l}-76(2 \cdot 1) \\
30 \\
0 \cdot 83(0 \cdot 22) \\
31 / 435 \\
0 \cdot 38(0 \cdot 08) \\
30 / 870 \\
3730(102) \\
20\end{array}$ \\
\hline
\end{tabular}

Mean (SE)

${ }^{a}$ Number of fibres studied

'Number of fibres/number of potentials counted

$\star$ Significance $p<0.01$ from normals

MEPP-miniature endplate potential

ACh-Acetylcholine
No impulse blocking was observed. Fibre densities were normal. No spontaneous fasciculations or denervation potentials were observed.

\section{Endplate physiology}

Intracellular recordings at the endplate region of intercostal muscle fibres revealed that the resting membrane potentials were similar to controls (table 2). Spontaneous miniature endplate potential (mepp) frequency was reduced $(p<0.01)$ compared with controls. Mean mepp amplitude was reduced $(p<0.01)$ compared with control values. The shape of the mepps was slightly altered as the potentials were prolonged by $20 \%$. As many of the mepps were small $(<0.5 \mathrm{mV})$, it is possible that some were not observed above the noise level of the recording system accounting for the reduced frequency.

Microiontophoretic application of $\mathrm{ACh}$ to the endplate region revealed decreased and marked, mean (SD), variability of the ACh sensitivity in different fibres [2730 (533) $\mathrm{mV} / \mathrm{nC}$ to 448 (128)]. Mean ACh sensitivity was reduced significantly $(p<0.01)$ compared with controls (table 2). Falling phase of the ACh potential was prolonged by $20 \%$.

\section{Ultrastructure}

Endplate regions at low magnification showed that the subjunctional myofibrils appeared normal without evidence of a myopathy. Neuromuscular junctions contained numerous synaptic vesicles, mitochondria and synaptic clefts with well established basal lamina. The endplate soleplasm contained an increase of smooth endoplasmic reticulum, ribosomes, polysomes and invaginated nuclei compared with controls suggesting high metabolic and regenerative activity (figure 2 ). Some endplates showed subsynaptic vesiculation and phagocytic lysosomal activity suggesting degeneration. At higher magnification some of the secondary synaptic clefts were widened and filled with debris and junctional folds were simplified suggesting endplate damage.

\section{Discussion}

The patient described in this report had a subacute progressive neuromuscular syndrome associated with CNS dysfunction following the fifth weekly spraying with phosmet, an OP insecticide. Paralysis became maximum by 9 days, continued for 30 days, but some weakness persisted for 4 months. CNS signs of visual hallucinations, disorientation and myoclonic jerks occurred at the peak of the toxicity and most likely reflected central cholinergic dysfunction. These signs cleared rapidly without residual effects. Limited symptoms and signs of acute OP toxicity were noted. No myopathy or delayed peripheral neuropathy occurred. The neuromuscular dysfunction was asseciated with CNS involvement which was observed also in patients with the intermediate syndrome following exposure to OP insecticides. ${ }^{10}$ Acute and delayed CNS cholinergic dysfunction is well recognised in 


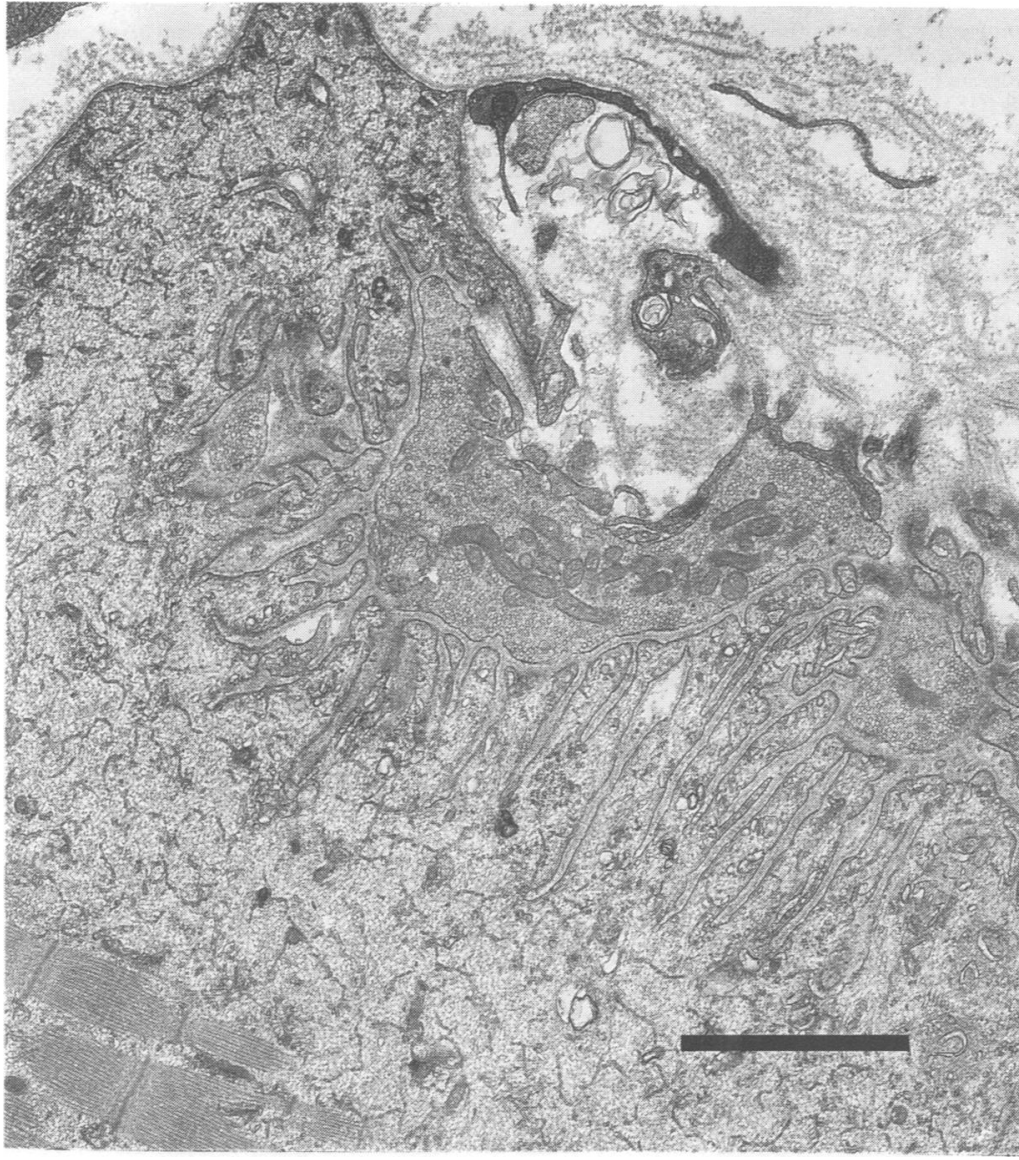

Figure 2 High magnification micrograph of a neuromuscular junction of intercostal muscle showing numerous synaptic vesicles and clefts. The soleplasm contains large amounts of smooth endoplasmic reticulum and polysomes. Calibration bar $=2 \mu \mathrm{m}$. experimental animals exposed to $\mathrm{OP}^{29}$ Although the paralysis in our patient did not occur after overt cholinergic toxicity, progressed over a longer period ( 9 days rather than 4) and persisted longer ( 30 days rather than 18) than those reported, ${ }^{10}$ it is likely that OP toxicity due to phosmet can produce a delayed progressive neuromuscular syndrome in humans. In this syndrome the endplate physiological studies clearly document postsynaptic neuromuscular dysfunction. This is characterised by: mildly reduced CMAP amplitudes, decremental responses reversed by edrophonium and neostigmine, small mepps and reduced junctional $\mathrm{ACh}$ sensitivity. These results support those of the previous report in which limited electromyographic studies suggested a postsynaptic defect. ${ }^{10}$ Decremental responses at slow rates of stimulation observed in this patient but not in those reported in the intermediate syndrome ${ }^{10}$ may reflect greater neuromuscular blockade, desensitisation and loss of ACh receptors. This may account for the prolonged duration of the syndrome. This subacute OP postsynaptic neuromuscular block can be differentiated electrophysiologically from acute OP cholinergic block that results in spontaneous repetitive firing of muscle action potentials and decremental-incremental responses. ${ }^{17}$ In acute OP toxicity, edrophonium increased the decremental responses, ${ }^{8}$ while in subacute toxicity it reduced the decremental responses toward normal. Edrophonium is recommended as a test in OP toxicity to document the acute cholinergic block. The acute syndrome may benefit from therapy with pralidoxime chloride, an AChE reactivator that may have varied actions, ${ }^{18}$ but it should not be used in the subacute syndrome with postsynaptic dysfunction, since it may increase the neuromuscular block and weakness.

The endplate physiological findings in this patient with subacute neuromuscular weakness are similar to those reported in myasthenia gravis that have shown loss of functional postsynaptic ACh receptors. ${ }^{15} 19$ The mechanism of receptor loss in subacute phosmet toxicity is unknown but is likely to result from endplate degeneration as shown on the ultrastructural studies. The physiological findings of mildly prolonged distal motor latency and reduced mepp frequency and the pathological finding of endplate damage suggest that nerve terminals may be affected by OP toxicity. Experimental studies with chronic AChE inhibitor toxicity in rats have shown similar physiological and morphological changes of the neuromuscular junctions. ${ }^{2021}$ Postsynaptic dysfunction was greater and persisted longer than presynaptic changes, was prevented by chronic nerve section and was therefore possibly due to the prolonged increased activity of synaptic $\mathrm{ACh}$. Prolonged action of $\mathrm{ACh}$ at the NMJ has been reported to produce a myopathy that is mediated by calcium activation of a protease and requires intact $\mathrm{ACh}$ receptors. ${ }^{22}$ A myopathy was not observed in our patient and this may reflect the endplate degeneration with loss of receptors. Prolonged action of synaptic ACh accounts for the acute OP syndrome, and it is possible that it may induce the subacute neuromuscular syndrome by prolonged receptor desensitisation and influx of calcium that damages the NMJ. The endplate damage may be enhanced also by the direct effects of $\mathrm{AChE}$ inhibitors that act as $\mathrm{AChR}$ agonists and channel blockers. ${ }^{23}$ It is possible that similar changes may occur at CNS cholinergic junctions. In addition, unpublished data from our laboratory suggest that organophosphates cause release of excitatory and inhibitory transmitters in the CNS. ${ }^{24}$

Our patient was exposed to phosmet, an insecticide recommended for the control of fruit tree pests. ${ }^{25}$ It is less toxic as an AChE inhibitor than other insecticides such as parathion. ${ }^{26}$ Phosmet has very low water solubility and is lipid soluble. ${ }^{26}$ It is possible that repeated weekly exposure to a lipid soluble $O P$, like phosmet, spray by inhalation and skin contact resulted in the subacute progressive neuromuscular and CNS syndrome in our patient. There are no other reports of subacute toxicity due to phosmet but fenthion, which also has high lipid solubility, produced 4 of the 10 reported cases of the intermediate syndrome. ${ }^{10}$ Proposed mechanisms by which some of the OP may produce subacute effects range from higher lipid solubility to delayed conversion to a more active metabolite. ${ }^{27}$ Although it is possible that the insecticide which the patient used contained a toxic product of phosmet, there are no reports of other toxic substances in the compound. ${ }^{25}$ 
Supported in part by the Neuromuscular Fund at the University of Maryland School of Medicine (RFM) and a Grant from the US Army Medical Research and Development Command Contract DAMD17-88-C-8119 (EXA).

1 Wadia RS, Sadagopan C, Amin RB, Sardesai HV. Neurological manifestations of organophosphorus insecticide poisoning. F Neurol Neurosurg Psychiatry 1974;37:841-7.

poisoning. F Neurol Neurosurg Psychiatry 1974;37:841-7.
2 Russell RW, Overstreet DH. Mechanisms underlying sensitivity to organophosphate anticholinesterase compounds. Prog Neurobiol 1987;28:97-129.

3 Namba T, Nolte CT, Jackrel J, Grob D. Poisoning due to organophosphate insecticides. Am $f$ Med 1971;50: 475-92.

4 Wadia RS, Chitra S, Amin RB, Kiwalkar RS, Sardesai HV. Electrophysiological studies in acute organophosphate poisoning. I Neurol Neurosurg Psychiatry 1987;50: 1442-8.

5 Albuquerque EX, Aracava $Y$, Idriss $M$, Schoenenberger B, Brossi A, Deshpande SS. Activation and blockade of the nicotinic and glutamatergic synapses by reversible and irreversible cholinesterase inhibitors. In: Dun NJ and Perlman RL, eds. Neurobiology and acetylcholine. New York: Plenum, 1987:301-28.

6 Albuquerque EX, Alkondon M, Deshpande SS, Cintra WM, Brossi A. The role of carbamates and oximes in reversing toxicity of organophosphorus compounds: a reversing toxicity of organophosphorus compounds: a perspective into mechanisms. In: Lunt GG, ed. Neurotox
'88: molecular basis of drug and pesticide action. Cambridge,
UK: Elsevier, 1988:349-73.

7 Albuquerque EX, Aracava Y, Cintra WM, Brossi A, Schoenenberger B, Deshpande SS. Structure-activity relationship of reversible cholinesterase inhibitors: activation, channel blockade and stereospecificity of the nicotinic acetylcholine receptor-ion channel complex. Brazilian $\mathcal{f}$ Med Biol Res 1988;21:1173-96.

8 Maselli R, Jacobsen JH, Spire J-P. Edrophonium: An aid in the diagnosis of acute organophosphate poisoning. Ann Neurol 1986;19:508-10.

9 Abou-Donia MB, Lapodula DM. Mechanisms of organophosphorus ester-induced delayed neurotoxicity: Type I and Type II. Ann Rev Pharmacol Toxicol 1990;30:405-40.

10 Senanayake N, Karalliede L. Neurotoxic effects of organophosphorus insecticides. An intermediate syndrome. $N$ phosphorus insecticides. An

11 Good JL, Khurana R, Mayer RF, Albuquerque EX. Endplate degeneration in organophosphate (Phosmet) poisoning. Neurology 1990;40(Suppl 1):431.

12 Mayer RF. Nerve conduction studies in man. Neurology (Minneap) 1963;13:1021-30.

13 Mayer RF, Williams IR: Incrementing responses in myasthenia gravis. AMA Arch Neurol 1974;31:24-6.

14 Stalberg E, Ekstedt J. Single fibre EMG and microphysiology of the motor unit in normal and diseased human muscle. In: Desmedt JE, ed. New development in electromyography and clinical neurophysiology. vol 1. Basel: Karger, 1973;113-29.

15 Albuquerque EX, Rash JE, Mayer RF, Satterfield JR. An electrophysiological and morphological study of the neuromuscular junction in patients with myasthenia gravis. Exp Neurol 1976;51:536-63.

16 Rash JE, Albuquerque EX, Hudson CS, Mayer RF, Satterfield JR. Studies of human myasthenia gravis: electrophysiological and ultrastructural evidence compatible with antibody attachment to the acetylcholine receptor complex. Proc Natl Acad Sci USA 1976;73:4584-8.
to

17 Besser R, Gutmann L, Dillmann U, Weilemann LS, Hopf HC. End-plate dysfunction in acute organophosphate intoxication. Neurology 1989;39:561-7.

18 Alkondon $M$, Albuquerque EX. The nonoxine bispyridinium compound SAD-128 alters the kinetic properties of the nicotinic acetylcholine receptor ion channel: A possible mechanism for antidotal effects. F Pharmacol Exp Ther 1989;250:842-52.

19 Cull-Candy SG, Miledi R, Trautmann A. End-plate currents and acetylcholine noise at normal and myasthenic human end-plates. 7 Physiol (Lond) 1979;287:247-65.

20 Tiedt TN, Albuquerque EX, Hudson CS, Rash JE. Neostigmine-induced alteration at the mammalian neuromuscular junction. I. Muscle contraction and electromuscular junction. I. Muscle contraction and electr

21 Hudson CS, Rash JE, Tiedt TN, Albuquerque EX. Neostigmine-induced alterations at the mammalian neuromuscular junction. II. Ultrastructure. $\mathcal{F}$ Pharmacol Exp Ther 1978;205:340-56.

22 Leonard J, Salpeter M. Agonist-induced myopathy at the neuromuscular junction is mediated by calcium. $f \mathrm{Cell}$ Biol 1979;82:811-9.

23 Pascuzzo GJ, Akaiki A, Maleque MA, Shaw K-P, Aronstam RS, Rickett DL, Albuquerque EX. The nature of the interactions of pyridostigmine with the nicotinic acetylcholine receptor-ionic channel complex. I. Agonist, desensitizing and binding properties. Mol Pharmacol 1984;25:92-101.

24 Pereira EFR, Aklondon M, Albuquerque EX. Effects of organophosphate compounds and physostigmine (PHy)
on nicotinic acetylcholine receptors (AChR) in the on nicotinic acetylcholine receptors (AChR) in the Medical Defense Bioscience Review 1991:229-33.

25 MacNeil J, Hikichi M. Phosmet residues in an orchard and adjacent recreational area. $\mathcal{f}$ Environ Sci Health 1986 21:375-85.

26 Dedek W, Grahl R, Schmidt R. A comparative study of guanine $\mathrm{N}$-alkylation in mice in vivo by the organophosphorus insecticides trichlorphon, dimethoate, phosmet, and bromophos. Acta Pharmacol et Toxicol 1984;55. 104-9.

27 Sakamoto T, Sawada Y, Kazuyuki N et al. Delayed neurotoxicity produced by an organophosphorus compound (Sumithion). Arch Toxicol 1984;56:136-8. 